\section{Cardiac and skeletal actin gene mutations are not a common cause of dilated cardiomyopathy}

EDITOR-Idiopathic dilated cardiomyopathy (DCM) is a primary disorder of heart muscle that is characterised by dilatation and impaired contraction of the chambers of the heart. Presentation is usually with heart failure, which is progressive, with a five year mortality of $50 \%$ after the onset of symptoms. Since the cause of this condition is unknown, specific therapy is not possible, and heart transplantation is the only definitive treatment for end stage disease.

A major advance in the study of the pathogenesis of DCM has been the demonstration that $20-30 \%$ of cases are familial, suggesting that genetic factors may be involved in the aetiology of the condition.' Reported families most commonly are compatible with autosomal dominant inheritance, but some with $\mathrm{X}$ linked and autosomal recessive inheritance have been documented. Genetic linkage studies in single large dominant pedigrees have implicated seven different chromosomal loci, underlining the genetic heterogeneity of the condition. ${ }^{2-4}$

Recently, missense mutations in the cardiac alpha actin gene that appeared to cosegregate with DCM were identified in two unrelated small families, raising the possibility that proteins involved in the generation or transmission of force in cardiac myocytes may be involved in the pathogenesis of DCM. ${ }^{5}$ The evidence in favour of a pathological role was confined to the conservation of the affected residues and the absence of the mutations from a large series of controls ( 870 chromosomes) (without unequivocal evidence of linkage or a functional deficit induced by the mutations). In addition, the prevalence of cardiac actin mutations in DCM is unknown. Furthermore, cardiac and skeletal actin genes are coexpressed in the heart, and the skeletal isoform, which is highly homologous in structure and function, is thought to be the predominant isoform in adult human hearts based on mRNA expression data. ${ }^{6}$ Protein quantitation studies have shown a lower but significant proportion of the skeletal actin isoform (20\%) compared to the cardiac actin isoform $(80 \%)$ in the adult human heart. ${ }^{7}$ We postulated that both cardiac and skeletal actin genes were important candidate genes for study in patients with dilated cardiomyopathy.

The patients and families were identified through a prospective multicentre study of the genetics of DCM in South Africa. Informed consent was obtained from each participant, and the study approved by the University of Cape Town Faculty of Medicine Research Ethics Committee. After exclusion of other causes of heart disease and known systemic disease, the diagnosis of DCM was based on the demonstration by echocardiography of left ventricular dilatation (left ventricular end diastolic diameter $>2.7$ $\mathrm{cm} / \mathrm{m}^{2}$ body surface area), and impaired systolic function (fractional shortening $<25 \%$ ). ${ }^{8}$ Cardiac catheterisation was performed in the majority of the patients to rule out coronary artery or other heart disease.

A total of 57 patients aged $1-79$ years (mean 40 years) with DCM were studied, including 38 males. The majority of the patients $(56 \%)$ were of black African origin. Eleven subjects from eight families had known familial disease with an autosomal dominant pattern of inheritance. All patients had a physical examination, ECG, and $2 \mathrm{D}$ transthoracic echocardiography including $M$ mode and Doppler studies. None of the patients had clinical evidence f Med Genet 1999;36:796-797 of skeletal muscle disease. All patients had left ventricular dilatation with a mean fractional shortening of $20 \%$ by echocardiography.

Genomic DNA isolated from peripheral leucocytes was amplified by PCR. The six exons of the cardiac alpha actin gene were amplified using published intronic primers; primers were designed for the amplification of the skeletal alpha actin gene exons according to the published genomic DNA sequence. ${ }^{9}$ The skeletal alpha actin gene primer sequences and the PCR product sizes are given in table 1. Note that primers are positioned in introns to include splice junctions and that exon 2 is amplified in two overlapping fragments of appropriate size for SSCP. The PCR products were analysed by SSCP performed on $6 \%$ polyacrylamide gels with and without addition of glycerol as previously described. ${ }^{\text {to }}$ Direct sequencing of abnormal SSCP conformers was performed using a standard protocol. ${ }^{10}$

Four single nucleotide variants (T315C, C431T, $\mathrm{C} 675 \mathrm{~T}$, and $\mathrm{C} 2918 \mathrm{~T}$ ) were found in the cardiac actin gene as outlined in fig $1 \mathrm{~A}$. The only variant in a coding region was a silent $\mathrm{C}$ to $\mathrm{T}$ transition affecting the third position of codon 73, C431T (Ile73Ile). The T315C and C675T variants are in intron 1 and intron 2 , respectively, and are sufficiently far from the splice sites not to have an effect on the transcript. The $\mathrm{C} 2918 \mathrm{~T}$ variant is in the 3 ' untranslated region of the cardiac actin gene and was present in $3 / 57$ cases and $2 / 60$ controls. Three variants were found in the skeletal actin gene (fig $1 \mathrm{~B}$ ). Two were silent transversions A2238C (Thr151Thr) and C3152A (Ile332Ile) in exons 2 and 6 respectively. A T $1910 \mathrm{C}$ transition at position -5 of the splice acceptor site in intron 1 was found in two subjects. As this substitution would not replace the consensus pyrimidine base at this position it would not be expected to affect splicing of the skeletal alpha actin gene transcript. ${ }^{11}$ Thus, none of the 57 patients (54 unrelated subjects) was found to have a mutation that was predicted to alter either the cardiac or skeletal actin peptides. The frequency of each of the cardiac and skeletal actin gene polymorphisms was $2-5 \%$ in the sample studied.

The main conclusion reached from this study is that actin mutations are not a common cause of DCM. Thus, although cardiac actin missense mutations have been described in two small families with DCM, their absence from 54 unrelated probands suggests that, at most, they could contribute a few percent of the disease prevalence. While the genetic background of our study population was clearly quite different from that of Olson et $a l^{5}$, previous experience suggests that dominant mutations associated with disease of this severity do not survive for long enough to create marked founder effects and so are likely to be evenly distributed among populations. ${ }^{12}$ On the other hand, the frequency of silent polymorphisms in this South African cohort (2-5\%) does contrast with that of the previous report (no polymorphisms identified) ${ }^{5}$ and this may reflect a population which is much older and had not been affected by recent expansion or bottlenecks.

Table 1 PCR primers for the amplification of the skeletal alpha actin gene exons $\left(5^{\prime} \rightarrow 3^{\prime}\right)$

\begin{tabular}{|c|c|c|c|}
\hline Exon & Fortard & Recerse & $\begin{array}{l}\text { PCR } \\
\text { product } \\
\text { (bp) }\end{array}$ \\
\hline 1 & TCGCGGTCTCCCTGTCCTTG & CCGAGCCGGCTCCCTCTGCG & 198 \\
\hline $2 \mathrm{i}$ & GGCGTTAACGGGTGCGTGGT & CGGTGAGCAGGGTGGGGTGC & 230 \\
\hline $2 \mathrm{ii}$ & TCTGGCACCACACCTTCTAC & CGGGACGAGGGGACTGGGGG & 229 \\
\hline 3 & СССССAGCCACTCACTCTCT & GCGGCCCTCCGCCCGGGTGC & 231 \\
\hline 4 & AGTGAGGGCTCCTCTCCTGC & GCCTGGGGGCCGGGGCGAGG & 262 \\
\hline 5 & GAGCTTCTGCTCACGCTCCC & CACCCCGCCGACAGCCCGCG & 251 \\
\hline 6 & GGTGAGGTCTCCCCACCTCA & GTCCTGAGAAGTCGCGTGCT & 213 \\
\hline
\end{tabular}




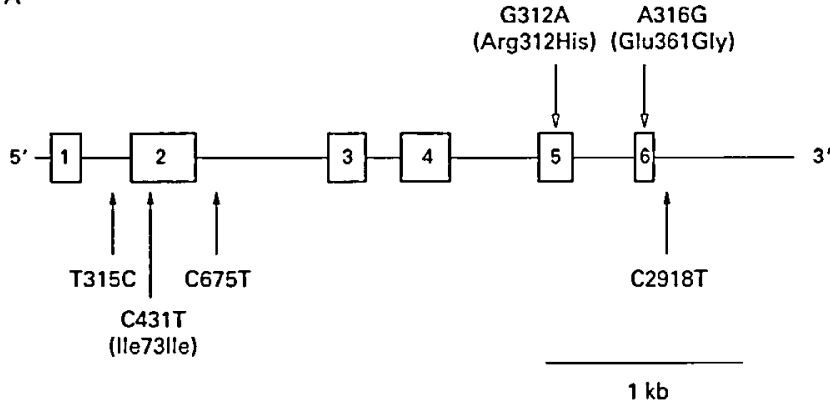

B

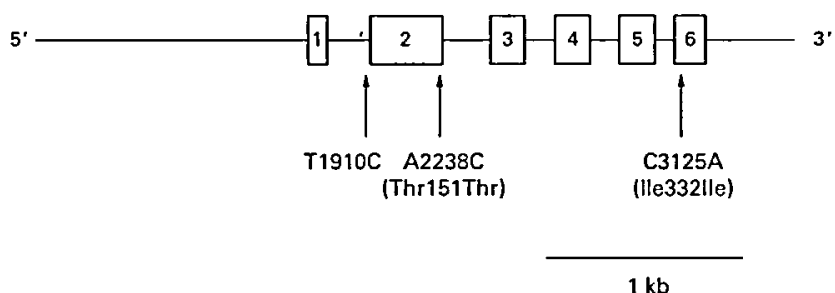

Figure 1 Schematic representation of the cardiac alpha actin gene $(A)$ and the skeletal alpha actin gene $(B)$. $(A)$ Location of the four single nucleotide variants found in this study (closed arrows) and the published cardiac alpha mutations associated with dilated cardiomyopathy. $(B)$ Location of the three single nucleotide variants found in the skeletal alpha actin gene.

We could not find support for the involvement of skeletal actin as a disease gene for DCM. However, if cardiac actin mutations cause DCM at a very low frequency, the same remains possible for the skeletal gene. The lack of an overt skeletal myopathy despite expression in skeletal muscle probably does not preclude skeletal actin as a candidate gene, as a comparable situation applies in hypertrophic cardiomyopathy caused by mutations in $\beta$-cardiac myosin heavy chain or $\alpha$-tropomyosin. The difficulty in defining or excluding a role for candidate genes in DCM relates to the anticipated extreme genetic heterogeneity, as indicated by the multiple anonymous loci in man and the diversity of mouse knockout models with a DCM phenotype. ${ }^{13}$ It remains unknown whether there are disease genes that are responsible for a significant proportion of this condition, but if there are these do not include those encoding cardiac or skeletal actin. BMI is a Nuffield Dominion Fellow; SK, BZ, and HW are supported by the Research Council of South Africa, the Medtronic Cardiovascular Institute of Routhern Africa, and the Cardiac Clinic Research Fund of the University of Southern Africa, and the Cardiac Clinic Research Fund of the University of
Cape Town. We gratefully acknowledge Drs K P Mokhobo, P Mntla, A Mitha, Cape Town. We gratefully acknowledge Drs K P Mokhobo, P Mntla, A Mitha, wer, $S \mathrm{Nel}, \mathrm{H}$ de Klerk, and PJ Commerford for referral of patients for this study. BONGANI MI MAYOSI SAIB S KHOGALI BAIPING ZHANG HUGH WATKINS

Department of Cardiovascular Medicine, University of Oxford, John Radcliffe Hospital, Oxford OX3 9DU, UK

1 Keeling PJ, Gang Y, Smith G, et al. Familial dilated cardiomyopathy in the United Kingdom. Br Heart f 1995;73:417-21.

2 Mestroni L, Giacca M1. Molecular genetics of dilated cardiomyopathy. Curr Opin Cardiol 1997;12:303-9.

3 Towbin JA. The role of cytoskeletal proteins in cardiomyopathy. Curr Opin Cell Biol 1998;10:131-9.

4 Jung $M$, Poepping I, Perrot A, Ellmer AE, Reis A, Osterziel KJ. A family with autosomal dominant dilated cardiomyopathy maps to a novel locus in chromosome 2. Circulation 1998;98(suppl):1-246.

5 Olson TM, Michels VV, Thibodeau SN, Tai YS, Keating MT. Actin mutations in dilated cardiomyopathy, a heritable form of heart failure. Science tions in dilated card $1998 ; 280: 750-2$.

6 Boheler KR, Carrier L, de la Bastie D, et al. Skeletal actin mRNA increases in the human heart during ontogenic development and is the major isoform of control and failing hearts. J Clin Imest 1991;88:323-30.

7 Vandekerckhove J, Bugaisky G, Buckingham M. Simultaneous expression of skeletal muscle and heart actin proteins in various striated muscle tissues and cells. F Biol Chem 1986;261:1838-43.

8 Richardson P, McKenna W, Bristow M, et al. Report of the 1995 World Health Organization/International Society and Federation of Cardiology Task Force on the Definition and Classification of cardiomyopathies. Circulation 1996;93:841-2.

9 Taylor A, Erba HP, Muscat GE, Kedes L. Nucleotide sequence and expression of the human skeletal alpha-actin gene: evolution of functional regulasion of the human skeletal alpha-actin gene

10 Zhang B, Ye S, Herrmann SM, et al. A functional polymorphism in the regulatory region of the gelatinase $B$ gene in relation to severity of coronary regulatory region of the gelatinase B gene in r
atherosclerosis. Circulation 1999;99:1788-94.

11 Shapiro $M B$, Senapathy P. RNA splice junctions of different classes of eukaryotes: sequence statistics and functional implications in gene expression. Nucleic Acids Res 1987;15:7155-74.

12 Watkins $H$, Thierfelder $L$, Anan $R$, et al. Independent origin of identical beta cardiac myosin heavy-chain mutations in hypertrophic cardiomyopathy. Am J Hum Genet 1993;53:1 180-5.

13 Leiden JM. The genetics of dilated cardiomyopathy - emerging clues to the puzzle. N Engl F Afed 1997;337:1080-1.

\section{Subclinical cognitive impairment in autosomal dominant "pure" hereditary spastic paraplegia}

EDITOR-The hereditary spastic paraplegias are characterised by progressive spasticity, predominantly affecting the lower limbs. They have been divided into "pure" or complicated forms. ${ }^{12}$ Four loci for autosomal dominant pure hereditary spastic paraplegia (ADPHSP) have been mapped, on chromosomes 2p (SPG4), 8q (SPG6), 14q (SPG3), and 15q (SPG6). ${ }^{1-3}$

Cognitive impairment may be present in apparently "pure" hereditary spastic paraplegia. Tedeschi et al" found subclinical cognitive deficits in seven patients with autosomal recessive or autosomal dominant pure hereditary spastic paraplegia, when compared to normal, unrelated controls. Webb and Hutchinson ${ }^{5}$ investigated 31 patients from 12 kindreds with ADPHSP (loci not described) and found subclinical cognitive impairment, apparently restricted to cases over 50 years old. In addition, the description of an SPG4 linked family with paraplegia complicated by subcortical dementia suggests that "pure" hereditary spastic paraplegia and hereditary spastic paraplegia complicated by frank dementia may be allelic disorders. ${ }^{6}$

As a pilot study, we investigated the presence of subclinical cognitive impairment in family members from four Welsh ADPHSP families (families 26, 27, 28, and 36) using the Mini-Mental State Examination. Diagnostic criteria and clinical details for these families have been described previously. ${ }^{7}$ None of the subjects had an existing diagnosis of overt dementia.

The three largest families, 26, 27, and 28 , were genotyped by PCR amplification at microsatellite markers flanking and within the relevant ADPHSP candidate regions (table 1). Family 36 was not genotyped because of its small size. For families 26 and 28 , two point lod scores do not support linkage to any of the four known loci, and multipoint linkage analysis generated lod scores of $<-2$ throughout all four candidate regions, formally excluding linkage. For family 27, two point and multipoint linkage 